\title{
Stochastic Pore Network Generation from 3D Rock Images
}

\author{
Z. Jiang • M. I. J. van Dijke • K. Wu • G. D. Couples • \\ K. S. Sorbie · J. Ma
}

Received: 28 December 2010 / Accepted: 9 June 2011 / Published online: 24 June 2011

(C) The Author(s) 2011. This article is published with open access at Springerlink.com

\begin{abstract}
Pore networks can be extracted from 3D rock images to accurately predict multiphase flow properties of rocks by network flow simulation. However, the predicted flow properties may be sensitive to the extracted pore network if it is small, even though its underlying characteristics are representative. Therefore, it is a challenge to investigate the effects on flow properties of microscopic rock features individually and collectively based on small samples. In this article, a new approach is introduced to generate from an initial network a stochastic network of arbitrary size that has the same flow properties as the parent network. Firstly, we characterise the realistic parent network in terms of distributions of the geometrical pore properties and correlations between these properties, as well as the connectivity function describing the detailed network topology. Secondly, to create a stochastic network of arbitrary size, we generate the required number of nodes and bonds with the correlated properties of the original network. The nodes are randomly located in the given network domain and connected by bonds according to the strongest correlation between node and bond properties, while honouring the connectivity function. Thirdly, using a state-of-the-art two-phase flow network model, we demonstrate for two samples that the rock flow properties (capillary pressure, absolute and relative permeability) are preserved in the stochastic networks, in particular, if the latter are larger than the original, or the method reveals that the size of the original sample is not representative. We also show the information that is necessary to reproduce the realistic networks correctly, in particular the connectivity function. This approach forms the basis for the stochastic generation of networks from multiple rock images at different resolutions by combining the relevant statistics from the corresponding networks, which will be presented in a future publication.
\end{abstract}

Z. Jiang $(\varangle) \cdot$ M. I. J. van Dijke $\cdot$ K. Wu · G. D. Couples $\cdot$ K. S. Sorbie · J. Ma Institute of Petroleum Engineering, Heriot-Watt University, Edinburgh EH14 4AS, UK e-mail: Zeyun.Jiang@ pet.hw.ac.uk

Z. Jiang

School of Mathematical Sciences, University of Electronic Science and Technology of China, Chengdu 610054, China 
Keywords Porous media - Pore-network - Pore characterisation - Stochastic network · Permeability $\cdot$ Multi-phase flow properties

\section{Introduction}

In recent years, much progress has been made in the prediction of multi-phase flow properties (capillary pressures and relative permeabilities) of porous media based around solutions of the (Navier) Stokes equations (NS), including Lattice-Boltzmann methods, in digital representations of the irregular pore space. Although these simulations may be considered as the ideal way of calculating the flow properties, there are currently two main problems with this approach. The first problem lies in obtaining digital representations, either through 3D imaging methods or through reconstruction from 2D images, which are sufficiently detailed and which simultaneously have a sufficiently large, representative volume. If this first problem could be overcome, then the second problem would be that current computation power is by far not capable of carrying out the simulations for multi-phase flow in a representative volume. Multi-phase flow calculations not only involve the NS equations, but also the equations for the evolution of the interfaces between the fluid phases. Admittedly, for some very homogeneous porous media and narrow ranges of pore size, the above approach could just be feasible, but for any realistic rock or soil sample that has heterogeneity at different length-scales, this is simply not feasible yet. In particular, carbonate rocks, containing nearly half of the world's oil reservoirs, typically have microporosity with pore sizes of less than one micron, as well as large vugs and fractures.

An attractive alternative, with longstanding credentials, is to represent the pore space by a network of nodes (larger pore bodies) connected by bonds (narrower pore channels) with effective geometrical and associated multi-phase flow properties (see, for example, the review by Blunt et al.2002). This can in principle overcome the second problem, as flow simulations in these models are not very computationally intensive, and computations on representative volumes are feasible in most cases. For relatively homogeneous materials, such as Berea and Fontainebleau sandstones, (relative) permeabilities calculated from pore networks are in good agreement with measurements (Valvatne and Blunt 2004; Ryazanov et al. 2009).

To overcome the first problem, one needs to integrate critical information from pore space representations at different length scales. One way of addressing this problem would be to build a pore network based on information from different length-scales. The aim of this article is to extract the essential information from a network corresponding to a pore space representation at a given length-scale and use this to generate networks of arbitrary size, in preparation for generating networks that contain information from different length-scales. An alternative approach to overcome the multi-scale issue is to reconstruct continuous pore space representations based on sedimentological and diagenetic information (Bakke and Øren 1997; Biswal et al. 2009). This process-based reconstruction is able to take into account multiple pore length scales, as, for example, encountered in carbonate rocks (Biswal et al. 2009), although the representative calculation of multi-phase properties will probably still require extraction of pore networks.

Many studies have attempted to capture the critically important characteristics of the pore space with respect to a range of flow and transport properties. From a mathematical point of view, the geometry and topology of the pore space are characterised by the Minkowski functionals of (pore) volume, surface area, curvature and connectivity (Vogel et al. 2010). The latter studies have established links between these Minkowski functionals and, in particular, single-phase permeability. However, the Minkowski functionals are not sufficient for 
the characterisation of the geometrical properties of a porous structure, which determine the distribution of two fluid phases, and some of these functionals are very sensitive to the resolution of the (digital) pore space. In this article, we consider the geometrical properties (such as length, volume and shape factor) of the elements of extracted pore networks, which have straight bonds with constant cross section and radially symmetric nodes, as well as the network connectivity (topology), thus implicitly taking the Minkowski functionals into account.

There are three commonly used methods to extract pore networks from images of porous media: the medial axis-based algorithm (Lindquist et al. 1996), the maximal ball algorithm (Silin et al. 2004; Al-Kharusi and Blunt 2007) and the Voronoi diagram-based algorithm (Delerue and Perrier 2002). The first approach is the most frequently used and has been improved significantly by Jiang et al. (2007) with an efficient method to identify pores unambiguously. The topology (connectivity) of the pore space is strictly preserved in the extracted network and some geometrical properties, such as inscribed radius, shape factor and hydraulic radius, are calculated from measuring pore cross-sectional perimeter and area. Other properties, such as node spatial position, node and bond volume, as well as node coordination number, are directly measured through image analysis of the original pore space (Jiang 2008).

Vogel and Roth (2001) proposed a method for stochastic network generation, based on a pore-size distribution and a connectivity function measured directly from the serial sections of undisturbed soil samples. The connectivity function, which is also used in this article, is based on the 3D Euler number depending on pore size. Vogel and Roth confirmed through relevant flow simulations that, at least for the silty top soil under consideration, the complexity of the corresponding network structure was sufficiently determined by these two parameters. The network model, consisting of cylindrical bonds only, was generated from a face-centred cubic grid with maximum coordination number 12. A key step was the adaptation of the network topology to match the newly defined connectivity function, which defines connectivity both within and between different size ranges of pores and which is also used in the present article. However, the limited choice of network parameters is likely to be insufficient for more complex materials, such as carbonate rocks, which have a variety of pore shapes and sizes, as well as complex correlations between different pore properties. Arns et al. (2004) introduced a similar method for generating stochastic networks, based on a regular lattice, although only the coordination number distribution, and not the connectivity function, was honoured. Furthermore, they had to randomise the regular lattice through perturbation of the node locations. They successfully applied their method to Fontainebleau sandstone samples to demonstrate the effect of different types of topology (disordered, long bonds, coordination number distribution etc.) on relative permeablities.

Idowu and Blunt (2008) proposed a new method for stochastic generation of the networks of arbitrary size based on pore- and throat-size distributions and connectivity. They took into account not only the geometric properties of individual pores and throats, but also the correlation between radii of pores and connecting throats, as well as between pore volume and pore coordination number. As indicated by Sok et al. (2002), such correlations have a strong impact on multi-phase flow. However, the approach ignored the correlations of properties of individual network elements and, more importantly, it only considered the coordination number as a measure of network connectivity. Moreover, the calculated relative permeabilities for the original network and for equivalent stochastic networks did not match very well. It has become evident that global pore topology (connectivity), i.e. the correlation between pore size and pore topology, is crucial for prediction of multi-phase flow properties and the corresponding residual saturations (Sok et al. 2002). 
To overcome the above limitations, we propose a new methodology for characterisation of the extracted network and the subsequent generation of equivalent stochastic networks. In Sect. 2 we describe the extraction of the pore network from a 3D rock image and its statistical analysis, including the correlations between different properties of one element and between properties of different network elements, as well as the connectivity function. In Sect. 3, we present the generation of a random network based on the statistics, in particular, the linking of nodes by bonds based on both coordination number distribution and connectivity function. In Sect. 4, we validate the statistical description and stochastic network generation methods, based on two original networks extracted from the X-ray CT images of two rock samples. First, we assess if the properties of stochastically generated network models of the same size as the original are faithfully reproduced. Then, we investigate how the flow properties of generated networks of different sizes compare with the originally sized network. Finally, we address the representativity of the original network.

\section{Pore Network Characterisation}

\subsection{Network Extraction}

From a 3D voxelated rock image (e.g. a micro-CT image), we partition the pore space as a network of nodes connected by bonds. These extracted or original networks will be used to generate stochastic networks that have equivalent microscopic structures and similar macroscopic flow properties. Although our network extraction approach accurately reproduces most geometrical and topological (GT) characteristics of the underlying pore space, a number of assumptions are made, which affect the (choice of) properties of the extracted networks. Therefore, we start with a summary of the extraction method indicating the properties that are assigned to the network and the assumptions that are made in this process. The extraction method proceeds as follows (Jiang et al. 2007; Jiang 2008):

- Remove isolated pores and floating grain particles from the image.

- Obtain a topology-preserving skeleton or medial axis using a Euclidean distance-based thinning algorithm.

- Consider skeleton junctions as node centres and identify all the skeleton voxels connected to a node centre as belonging to a node backbone.

- Remove all node backbones from the medial axis and identify connected components consisting of the remaining skeleton voxels as bond backbones. This approach is topology-preserving and prevents the so-called snow-balling (Sheppard et al. 2005; Silin et al. 2004; Al-Kharusi and Blunt 2007; Lindquist et al. 2000 etc.).

- Assume radially symmetric nodes and straight bonds with a constant cross section. This requires the choice of properties for nodes and bonds as indicated in Fig. 1.

- Measure the geometrical properties of the nodes and bonds through digital image analysis.

- Further idealise the network model by removing redundant bonds between two nodes and creating virtual nodes for dead-end pores.

As indicated above, the network elements (nodes and bonds) are described by a set of geometric properties, whereas the network connectivity is characterised by the coordination number and the connectivity function, which is described in Sect. 2.2.3. In our network model (Ryazanov et al. 2009), we consider (inscribed) radius (R), volume (V) and shape factor (S) for each node $(\mathrm{N})$ and each bond $(\mathrm{B})$, coordination number $(\mathrm{C})$ for each node and length (L) 


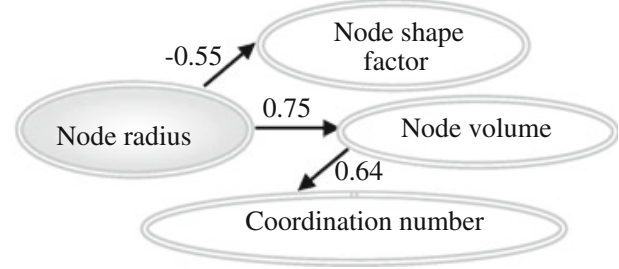

(a)

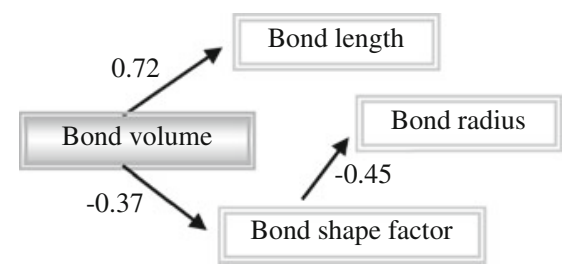

(b)

Fig. 1 Properties and dependence structure of network elements: primary properties (radius for nodes, volume for bonds) and the dependence structure of nodes (a) and bonds (b) for a Berea sandstone network

for each bond (see Fig. 1). In addition, idealised shapes, such as circles, triangles and regular stars, with shape factors approximating the extracted values, are used (Ryazanov et al. 2009).

\subsection{Statistical Pore Network Characterisation}

To characterise the pore network statistically, we need to describe its geometry and topology in terms of probability distributions for individual properties (volume, radius, shape factor etc.) of network elements and correlations between them. Based on the statistics, we first identify the most important, primary properties and quantify those using empirical probability distributions. Then, we determine correlations between primary and other properties based on the correlation strength and quantify the strong correlations with regression models and the weaker ones with conditional probability distributions. In addition, we quantify the pore space connectivity.

\subsubsection{Correlation-based Definitions}

There are two types of correlations in a pore network. One is between different properties of a single network element and the other is between a property of a node and a property of a bond, connected to that node. The former is referred to as an internal correlation and the latter as an external correlation. External correlations are based on a given property of a bond and the average of the values for a given property of the two adjoining nodes. In Fig. 2, examples are given of an internal correlation between node volume (NV) and node radius (NR), and an external correlation between NR and bond radius (BR) for the Berea sandstone network that will be used for validation in Sect. 4.

Note that we base the statistics on the interior nodes and bonds only. Since all the node centres are located strictly in the interior of the network domain, all the nodes are assumed to be interior. However, boundary bonds connecting nodes with the inlet or outlet faces are taken separately, and the connections with the corresponding nodes are not considered in the coordination numbers for the latter. We expect this to be a minor assumption for a sufficiently large network.

Correlation Coefficient: The correlation strength between two random variables $S$ and $T$ is measured through the correlation coefficient $\rho(S, T)$ (Pearson 1920; Jensen et al. 2000), which is the degree to which there is a linear relationship between the two variables. For several commonly used nonlinear relationships, transformations into linear models can be found, which will be given later on in this article. Having an observed sequence $\left\{\left(s_{1}, t_{1}\right), \ldots,\left(s_{n}, t_{n}\right)\right\}$ of $S$ and $T, \rho$ is estimated as (Jensen et al. 2000) 
(a)

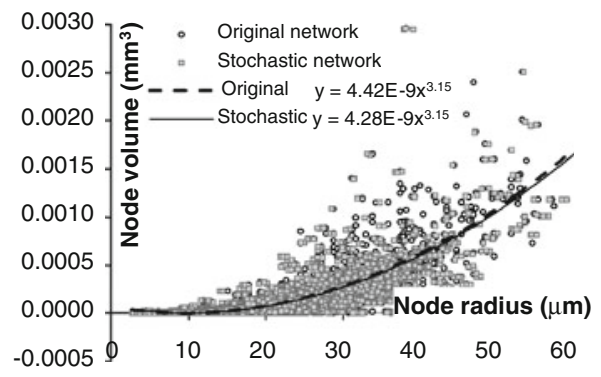

(b)

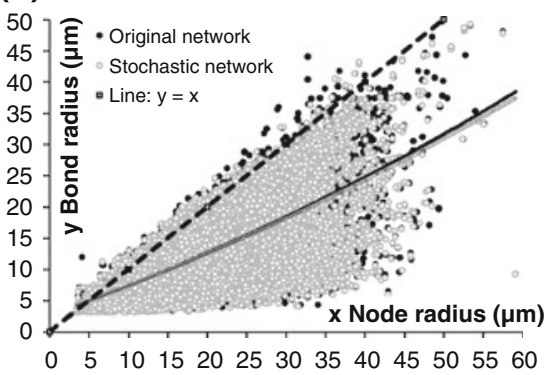

(c)

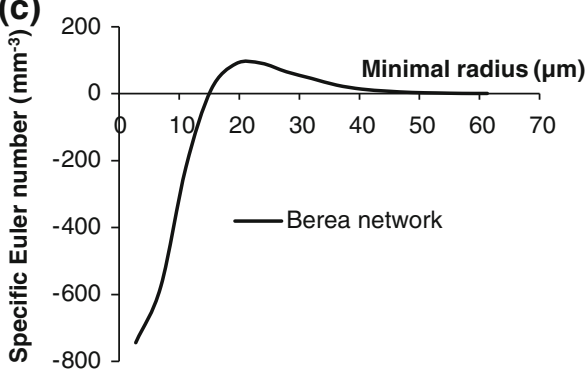

Fig. 2 The strong internal correlation a between node volume and node radius, the strong external correlation $\mathbf{b}$ between node radius and bond radius and $\mathbf{c}$ the connectivity function for the network extracted from a Berea sandstone sample. In subfigures $\mathbf{a}$ and $\mathbf{b}$ the same correlations are shown for stochastically generated networks of the same size as the original

$$
r_{(S, T)}=\frac{n \sum_{i=1}^{n} s_{i} t_{i}-\sum_{i=1}^{n} s_{i} \sum_{i=1}^{n} t_{i}}{\sqrt{n \sum_{i=1}^{n} s_{i}^{2}-\left(\sum_{i=1}^{n} s_{i}\right)^{2}} \sqrt{n \sum_{i=1}^{n} t_{i}^{2}-\left(\sum_{i=1}^{n} t_{i}\right)^{2}}} .
$$

For each type of network element (i.e. node or bond), we use the correlation coefficient to define one of the properties as being primary and to determine the dependence of the remaining properties on the primary property.

Primary property and dependence structure: Let $X_{1}, \ldots, X_{n}$ be $n$ properties of the node (bond) $X$, and $Y_{1}, \ldots, Y_{m}$ be $m$ properties of the bond (node) $Y$,

- Determine the strongest internal correlation, i.e. $\max _{i \neq j}\left|\rho\left(X_{i}, X_{j}\right)\right|$. If the sum of the remaining correlations for $X_{i}$ is greater than that for $X_{j}, \Sigma_{k \neq i}\left|\rho\left(X_{i}, X_{k}\right)\right| \geq \Sigma_{l \neq j} \mid$ $\rho\left(X_{j}, X_{l}\right) \mid$, then $X_{i}$ is the primary property, $X_{j}$ depends on $X_{i}$ and both $X_{i}$ and $X_{j}$ are now part of the dependence structure for $X$.

- Determine the strongest internal correlation between the properties $X_{i}$ in the dependence structure and the remaining properties $X_{j}$, i.e. $\max _{i \neq j}\left|\rho\left(X_{i}, X_{j}\right)\right|$, and add the resulting property to the dependence structure, until no more properties remain.

- Repeat the previous step until all properties of $X$ are added to the dependence structure.

- Record the strength of all correlations in the dependence structure.

- Determine the strongest external correlation, i.e. $\max _{i, j}\left|\rho\left(X_{i}, Y_{j}\right)\right|$. This will be used to connect nodes and bonds in the resulting stochastic network.

As an example, Table 1 lists all internal and external correlations for the Berea sandstone network. The dependence structure for the nodes and bonds in the Berea sample is as shown in Fig. 1. Note the following: In general, the internal correlations for nodes are stronger than 
Table 1 Correlation coefficients between different properties for a Berea sandstone (a) and a Castlegate sandstone (b) including both internal (highlighted) and external correlations

\begin{tabular}{|c|c|c|c|c|c|c|c|c|}
\hline & NR & NV & NS & $\mathrm{NC}$ & BR & BV & BS & BL \\
\hline \multicolumn{9}{|l|}{ (a) } \\
\hline NR & 1.00 & 0.75 & -0.55 & 0.58 & 0.70 & 0.56 & -0.51 & 0.32 \\
\hline NV & 0.75 & 1.00 & -0.40 & 0.64 & 0.50 & 0.48 & -0.34 & 0.23 \\
\hline NS & -0.55 & -0.4 & 1.00 & -0.36 & -0.43 & -0.28 & 0.42 & -0.16 \\
\hline $\mathrm{NC}$ & 0.58 & 0.64 & -0.36 & 1.00 & 0.30 & 0.29 & -0.23 & 0.18 \\
\hline $\mathrm{BR}$ & 0.70 & 0.50 & -0.43 & 0.30 & 1.00 & 0.33 & -0.45 & -0.10 \\
\hline BV & 0.56 & 0.48 & -0.28 & 0.29 & 0.33 & 1.00 & -0.37 & 0.72 \\
\hline BS & -0.51 & -0.34 & 0.42 & -0.23 & -0.45 & -0.37 & 1.00 & -0.23 \\
\hline $\mathrm{BL}$ & 0.32 & 0.23 & -0.16 & 0.18 & -0.10 & 0.72 & -0.23 & 1.00 \\
\hline \multicolumn{9}{|l|}{ (b) } \\
\hline NR & 1.00 & 0.76 & -0.44 & 0.67 & 0.59 & 0.42 & -0.36 & 0.20 \\
\hline NV & 0.76 & 1.00 & -0.34 & 0.75 & 0.40 & 0.34 & -0.24 & 0.14 \\
\hline NS & -0.44 & -0.34 & 1.00 & -0.31 & -0.33 & -0.19 & 0.30 & -0.07 \\
\hline $\mathrm{NC}$ & 0.67 & 0.75 & -0.31 & 1.00 & 0.28 & 0.28 & -0.18 & 0.18 \\
\hline BR & 0.59 & 0.40 & -0.33 & 0.28 & 1.00 & 0.20 & -0.29 & -0.23 \\
\hline $\mathrm{BV}$ & 0.42 & 0.34 & -0.19 & 0.28 & 0.20 & 1.00 & -0.32 & 0.74 \\
\hline BS & -0.36 & -0.24 & 0.30 & -0.18 & -0.29 & -0.32 & 1.00 & -0.18 \\
\hline BL & 0.20 & 0.14 & -0.07 & 0.18 & -0.23 & 0.74 & -0.18 & 1.00 \\
\hline
\end{tabular}

for bonds. In addition, there is a strong correlation between bond radius (BR) and node radius (NR). Unsurprisingly, the largest coefficient is reached for the correlation between NR and node volume (NV), hence NR is selected as the primary node property. NR has on average the strongest correlations with the other node properties, although the coordination number (NC) is most strongly correlated to NV. The dependence structure of the nodes starts with the primary property NR. Both node shape factor (NS) and NV depend directly on NR, while $\mathrm{NC}$ is indirectly determined by NR via NV. For bonds, BV has the strongest internal correlations, and hence, it is selected as the primary bond property. In the dependence structure of the bonds, BV strongly determines BL and moderately determines BS, which subsequently determines BR. Table 1 indicates that the strongest external correlation occurs between NR and BR.

The correlation strengths, i.e. the absolute values of the correlation coefficients in the dependence structure, determine how the relations between the various properties are described.

Correlation Categories: Given two constants $\alpha$ and $\beta(0<\alpha<\beta<1)$, all correlations can be divided into three categories, based on the correlation strength $|\rho(S, T)|$ between two properties $S$ and $T$ as follows (Rodgers and Nicewander 1988):

(1) for $|\rho(S, T)|<\alpha$, the correlation is weak and the corresponding relationship can be ignored;

(2) for $\alpha \leq|\rho(S, T)|<\beta$, the correlation is moderate, and the relationship will be described by a conditional probability distribution, as explained in the next section;

(3) for $|\rho(S, T)| \geq \beta$, the correlation is strong, and there may exist a linear (or transformed nonlinear) relationship given by a regression model. 


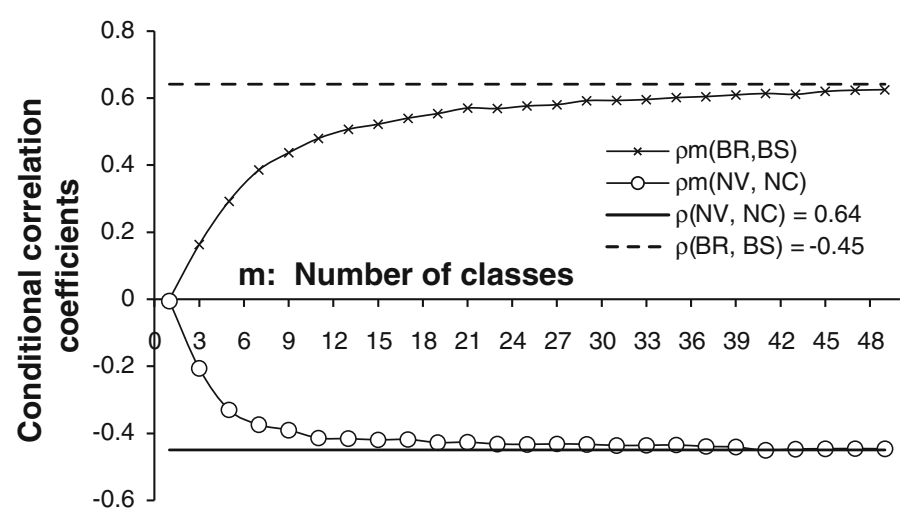

Fig. 3 Conditional correlation coefficients $\rho_{\mathrm{m}}$ for two correlations in the Berea sandstone network, which approach the original values $\rho$ as the number of classes increases

Following Jensen et al. (2000), we set $\beta=0.7$ in our implementation, but $\alpha$ is defined indirectly through the following definition.

Conditional Correlation Coefficients: Consider the set of observed pairs $\mathscr{M}=$ $\left\{\left(s_{1}, t_{1}\right), \ldots,\left(s_{n}, t_{n}\right)\right\}$, with $s_{1} \leq \cdots \leq s_{n}$, of two variables $S$ and $T$ with correlation coefficient $\rho(S, T)$, estimated using Eq. 1. Divide the observed values of $S$ in $\mathscr{M}$ into $m \leq n$ classes, say of equal intervals of $s$ values with length $\left(s_{n}-s_{1} / m\right)$, and let $\mathscr{M}_{\mathrm{si}}$ be the set of $s$ values and $\mathscr{M}_{\mathrm{ti}}$ the corresponding set of $t$ values for a given class $i=1, \ldots, m$. Now, randomly re-assign the $t$ values in $\mathscr{M}_{\mathrm{ti}}$ to the $s$ values in $\mathscr{M}_{\mathrm{si}}$ to create the randomised class $\mathscr{M}_{\mathrm{i}}^{\prime}$. Combine all randomised classes $\mathscr{M}_{\mathrm{i}}^{\prime}$ into the set of observations $\mathscr{M}^{\prime}$ and calculate the conditional correlation coefficient $\rho_{m}$.

Note that, for $m=1$, the entire observation set is randomised; hence, $\rho_{1} \approx 0$. On the other hand, the sequence of conditional correlation coefficients $\rho_{1}, \rho_{2} \ldots$ converge to the original correlation coefficient $\rho(S, T)$, since, for $m=n$, the set is not randomised. For the Berea sandstone, the curves of correlation coefficients, $\rho_{m}$ versus the number of classes $m$, are presented in Fig. 3 for two pairs of properties. Note that the properties BR and BS are weakly correlated in that $\rho_{m}$ is close to $\rho$ for only a few classes, and we may consider these properties as uncorrelated. The latter provides a criterion for the determination of the constant $\alpha$ that delineates the weak correlation category. On the other hand, the relatively strongly correlated NV and NC need many classes for $\rho_{m}$ to be close to $\rho$. Based on this curve, in the next section, a criterion will be set for the number of classes of the conditional probability distribution describing the relation between two properties.

\subsubsection{Statistical Descriptions}

Both primary properties and other independent properties (which are weakly correlated to all remaining properties) are described by their cumulative distribution function (CDF) or, equivalently, their probability distribution function (PDF). A strong relationship between a property $S$ and its dependant $T$ may be described by a regression equation or trend curve $T=\mathrm{f}(S)$, if available, using the least-squares method (Jensen et al. 2000). Note that a number of nonlinear regression equations can be converted into linear ones, as indicated in Table 2 for five commonly occurring nonlinear models. In the transformed linear regression model $T^{\prime}=f^{\prime}\left(S^{\prime}\right)+\varepsilon^{\prime}$, the random error $\varepsilon^{\prime}$ is assumed to be normally distributed, $\varepsilon^{\prime} \sim N\left(0, \sigma^{\prime}\right)$, 
Table 2 Linear and nonlinear models and their transformations commonly used in regression

\begin{tabular}{lll}
\hline Nonlinear model & Transformation & Linear model \\
\hline$y=b_{0}+b_{1} x^{1}+\cdots+b_{p} x^{p}$ & $x_{i}^{\prime}=x^{i}, i=1, \ldots, p$ & $y=b_{0}+b_{1} x_{1}^{\prime}+\cdots+b_{p} x_{p}^{\prime}$ \\
$\frac{1}{y}=a+b \frac{1}{x}$ & $y^{\prime}=\frac{1}{y}, \quad x^{\prime}=\frac{1}{x}$ & $y^{\prime}=a+b x^{\prime}$ \\
$y=a x^{b}$ & $y^{\prime}=\log y, \quad x^{\prime}=\log x, \quad a^{\prime}=\log a$ & $y^{\prime}=a^{\prime}+b x^{\prime}$ \\
$y=a e^{b x}$ & $y^{\prime}=\log y, \quad a^{\prime}=\log a$ & $y^{\prime}=a^{\prime}+b x$ \\
$y=a+b \log x$ & $x^{\prime}=\log x$ & $y=a+b x^{\prime}$ \\
\hline
\end{tabular}

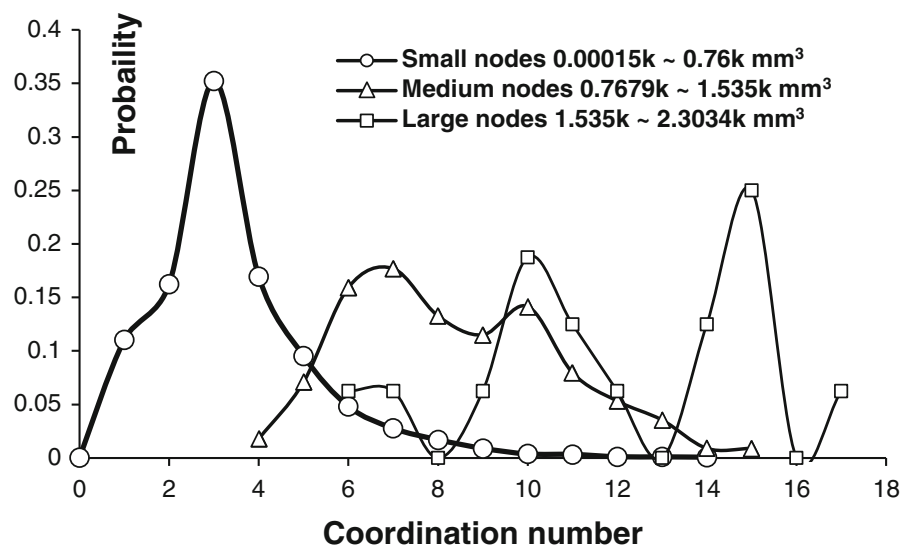

Fig. 4 A conditional probability distribution (CPD) consisting of three PDFs for the coordination number (NC), for small, medium and large node volumes (NV) respectively

where for the pairs of transformed observed values $\left\{\left(s_{1}^{\prime}, t_{1}^{\prime}\right), \ldots,\left(s_{n}^{\prime}, t_{n}^{\prime}\right)\right\}$ of $S^{\prime}$ and $T^{\prime}$, the standard deviation $\sigma^{\prime}$ is estimated as

$$
\sigma^{\prime} \approx \sqrt{\frac{\sum_{i=1}^{n}\left(t_{i}^{\prime}-f^{\prime}\left(s^{\prime} i\right)\right)^{2}}{n}}
$$

In Fig. 2a, the strong correlation between NR and NV for the Berea sample is given by a power law with exponent 3.15. The deviation for the untransformed data of NV ranges from $0.000012 \mathrm{~mm}^{3}$ to $0.00057 \mathrm{~mm}^{3}$ for the smallest and the largest radii, respectively.

Dependencies between variables $S$ and $T$ with moderate correlations or strong correlations for which no suitable regression models are available are described by conditional probability distributions (CPD), similar to the probability field simulation (Coburn et al. 2007). The CPD is related to the conditional correlation coefficients of Sect. 2.2.1, where the set $\mathscr{M}_{\mathrm{S}}$ of observed values of $S$ is divided into $m$ number of (equally sized) classes of $s$ values $\mathscr{M}_{\mathrm{si}}$, with the corresponding set of $t$ values $\mathscr{M}_{\mathrm{ti}}$. Then, for each class, $i=1, \ldots, m$, the probability distribution of the $t$ values in $\mathscr{M}_{\mathrm{ti}}$ is determined. Consequently, a CPD consists of a set of probability distributions (CDFs or PDFs) that are conditional to classes of $S$. From Fig. 4, note that the CPD is able to reveal the dependence of NC upon NV as the distribution curves tend to shift from to the right, indicating that larger nodes have more connections. 
To determine how many classes $m$ of $S$ for $T$ should be used, we consider the curve of the conditional correlation correlations, for which examples are shown in Fig. 3. Then, choose a sufficiently small 'tolerance' $\tau>0$ and choose $m$ such that $\left|\frac{\rho_{m}-\rho}{\rho}\right| \leq \tau<\left|\frac{\rho_{m-1}-\rho}{\rho}\right|$.

For example, Table 1a and Fig. 3 show a correlation strength $|\rho(N V, N C)|=0.64$ for node volume and node coordination number. Using $\tau=0.01$, we find that $m=47$ for the corresponding CPD. The chosen value for $\tau$ represents a practical balance between accuracy and computational efficiency.

\subsubsection{Pore Connectivity}

The connectivity of the pore space plays an important role in rock hydraulic properties and their hysteretic behaviour (Vogel and Roth 2001; Jiang et al. 2010). The local connectivity is given by the node coordination number $\mathrm{NC}$, which is defined as the number of bonds connected to a given node. Nevertheless, the NC distribution and its statistical properties (i.e. mean and standard deviation) do not provide sufficient global topological information about the pore structure. On the other hand, the specific Euler number $\chi_{v}$, for a given rock volume $V$, is widely considered as a suitable measure of average connectivity or topology for a rock as a whole (Vogel and Roth 2001) and is defined as

$$
\chi_{v}=\frac{N-C+H}{V} .
$$

The Euler number $\chi$ is the number of isolated objects (components) $N$ minus the number of redundant connections (tunnels) $C$ plus the number of completely enclosed cavities (isolated solid particles floating in the pore space) $H$. Jiang (2008) introduced an efficient method to compute the Euler number for a binary image, which is based on the concept of the topological number presented by Bertrand (1994).

However, the Euler number is unable to reveal the detailed pore space connectivity. To solve this problem, Vogel and Roth (2001) introduced the connectivity function (see Fig. 2c), which is defined as the specific Euler number calculated for the reduced pore space of pore size (radius) equal to or larger than a given value. This quantity provides information of pore connectivity both within and between different classes of pores. By removing levels of the smaller sized pores step by step, the connectivity gradually decreases (and the specific Euler number increases), until a globally unconnected state is reached (and the specific Euler number is positive).

Note that in a network, there are no isolated solids (i.e. floating particles in pore space), and hence, $H=0$ in Eq. 3, and it can be shown easily that the connectivity function for a network is simply computed as

$$
\chi_{V}(r)=\frac{N_{N}(r)-N_{B}(r)}{V},
$$

where $N_{N}(r)$ is the number of nodes and $N_{B}(r)$ the number of bonds with radii larger than or equal to $r$ (Vogel and Roth 2001), where each bond for $N_{B}(r)$ is only used to connect nodes that are counted for $N_{N}(r)$. 


\section{Stochastic Network Generation}

In this Section, we describe how a network is generated based on the statistical description and the dependence structure of the geometrical properties and coordination number, while honouring the connectivity function.

\subsection{Generate Random Property Values}

First, we discuss how to assign values for the various node and bond properties based on the statistical descriptions.

Random values from a probability distribution: As discussed in Sect. 2.2.2, values are generated directly from the cumulative distribution function (CDF) for both primary properties and for properties that have weak dependencies. For a continuous (strictly monotonic) CDF $F_{T}(t)$ of a variable $T$, a random value $t$ of $T$ is easily generated as follows:

- generate a random number $u$ from the Uniform distribution $U(0,1)$ (Jensen et al. 2000), and

- calculate $t=F_{T}^{-1}(u)$.

For discrete (not invertable) CDFs, we take for $F_{T}^{-1}$ the quasi-inverse of the CDF, defined as (Strelen and Nassaj 2007)

$$
F_{T}^{-1}(\mu)=\left\{\begin{array}{l}
\inf \{z \mid F(z) \geq \mu\}, \mu>0 \\
\sup \{z \mid F(z)=0\}, \mu=0
\end{array} .\right.
$$

Random values from a regression equation: If $T$ strongly depends on $S$ and a linear (or transformed nonlinear) regression model $T^{\prime}=f^{\prime}\left(S^{\prime}\right)$ is available (see Sect. 2.2.2), for a given value $s^{\prime}$ of $S^{\prime}$, a random number $\varepsilon^{\prime \prime}$ is generated from $N\left(0, \sigma^{\prime}\right)$, and $t^{\prime}=f^{\prime}\left(s^{\prime}\right)+\varepsilon^{\prime \prime}$ becomes the corresponding value of $T^{\prime}$.

Random values from a conditional probability distribution: If $T$ moderately depends on $S$ (or no regression model is available), then the conditional probability distribution (CPD) with $m$ classes of $S$ for $T$ is used, as described in Sect. 2.2.2. For a given value, s, of $S$, the corresponding conditional CDF of $T$ from the CPD is determined, and a random value $t$ of $T$ is generated from that CDF using its quasi-inverse (Eq. 5).

\subsection{Network Generation Algorithm}

Based on the statistical information of an original network, we generate an equivalent network of arbitrary size, as summarized in the following steps:

1. Choose the domain $\Omega$ for the stochastic network with dimensions ( $L, W, H$ ) (Fig. 5a).

2. Create the appropriate number of nodes, based on the scaling of the domain, and generate random values for each node as its radius, volume, shape factor and coordination number according to the statistics.

3. Randomly position the node centres in the domain $\Omega$, while avoiding overlap of the actual nodes (Fig. 5a).

4. Create the appropriate numbers of internal and boundary bonds and generate random values for their radii, volumes, lengths and shape factors.

5. Position the boundary bonds between the inlet or outlet faces and nodes, based on bond lengths (relative to the distances between the respective boundary and the node centres), (Fig. 5b). 

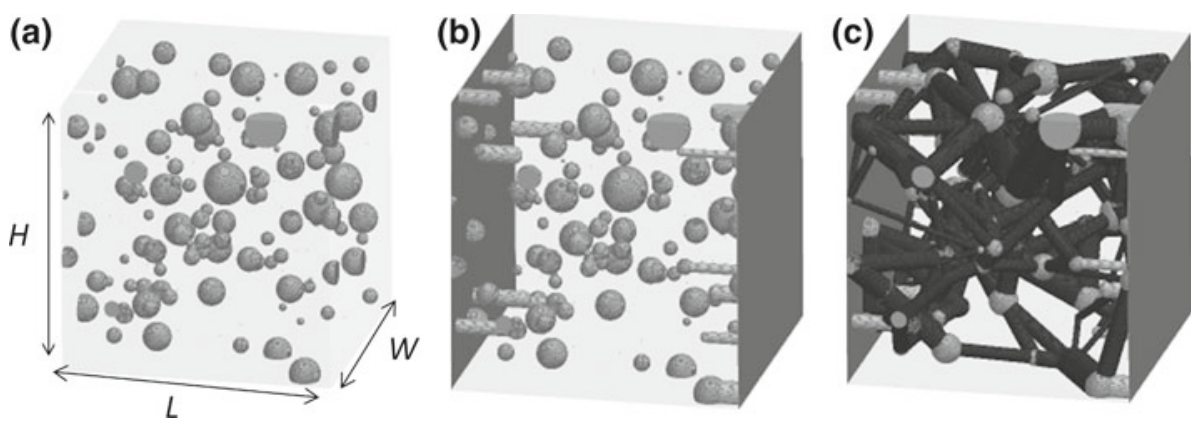

Fig. 5 Schematic steps for stochastic network generation: a randomly locate nodes in a desired domain of dimensions $(L, W, H)$, b insert boundary bonds to build up external connections to the inlet and outlet, $\mathbf{c}$ create internal connections by positioning bonds according to the strongest external correlation and the connectivity function

6. Position the bonds between pairs of nodes with implementation of the strongest external correlation (Fig. 5c), while observing the connectivity function and the bond lengths (relative to the distances between nodes).

Based on an original network of dimensions $(l, w, h)$, we set the dimensions $(L, W, H)$ of the stochastic network using a scaling factor $\xi>0$, such that $L=\xi l, W=\xi w$ and $H=\xi h$. Given the number of nodes $N_{n}$ in the original network, with volume $V=l w h$, the number of nodes $N_{n}^{\prime}$ in the stochastic network is simply scaled with its volume $V^{\prime}=\xi^{3} V$, i.e.

$$
\mathbf{N}_{n}^{\prime}=\xi^{3} \mathbf{N}_{n} .
$$

Because we did not obtain statistics on the spatial distribution of the nodes, the locations of the node centres are randomly chosen in the $3 \mathrm{D}$ domain $\Omega$. However, before a new node $k$ with radius $r_{k}$ is put in place, we make sure that it does not overlap with already existing nodes $j(j=1, \ldots, k-1)$ by checking that

$$
r_{k}+r_{j}>d_{k j}
$$

where $d_{j k}$ is the Euclidean distance between nodes, $j$ and $k$. If inequality (7) is not satisfied, then a new location for node $k$ is randomly chosen. For computational efficiency, the detection of overlap is only carried out for node centres in a sub-domain of $\Omega$ containing the new node and in adjacent sub-domains. Obviously, the diameter of each sub-domain must at least be equal to the sum of radii for all pairs of nodes, $\max _{k, j}\left(r_{k}+r_{j}\right)$. A similar, more general, overlap detection algorithm was proposed by Biswal et al. (2009).

Assuming that the specific Euler number $\chi_{v}$ (Eq. 4) is the same for the original and stochastic networks, similar to Eq. 6 the number of (interior) bonds $N_{b}$ scales trivially as

$$
N_{b}^{\prime}=N_{n}^{\prime}-\chi_{V} \xi^{3} V=\xi^{3} N_{b},
$$

The number of boundary bonds $N_{b b}$, which connect nodes with the inlet or outlet faces, scale as $N_{b b}^{\prime}=\xi^{2} N_{b b}$, as they appear only in these 2D planes.

To position the bonds, we proceed as follows. Let $O$ be the set of nodes with the stochastically generated properties and $\Theta$ the set of generated (interior) bonds. We equally divide the range of radii of all network elements, $r_{\min }$ to $r_{\max }$ into a sufficiently large number of $m-1$ intervals separated by $r_{\min }=r_{1}<\cdots<r_{m}=r_{\max }$, and obtain the corresponding specific Euler numbers (values of the connectivity functions), $\chi_{v}\left(r_{1}\right) \ldots \chi_{v}\left(r_{m}\right)$, from the original 
network. Furthermore, let $P_{n}$ and $P_{b}$ be, respectively, the node and bond properties with the strongest external correlation (e.g. $P_{n}$ is NR and $P_{b}$ is BR in Fig. $2 \mathrm{~b}$ for the Berea network).

Starting from the largest elements, select for each $k=m-1, \ldots, 1$, the set of $N_{n}\left(r_{k}\right)$ nodes $O_{k}$ from $O$ with node radius NR $\geq r_{k}$ and the set of $N_{b}\left(r_{k}\right)$ bonds $\Theta_{k}$ from $\Theta$ with bond radius $\mathrm{BR} \geq r_{k}$. Determine the number $N_{b k}=N_{n}\left(r_{k}\right)-\chi_{v}\left(r_{k}\right) V^{\prime}$, according to Eq. 4 , where $V^{\prime}$ is the volume of the domain $\Omega$. If $N_{b}\left(r_{k}\right)>N_{b k}$ randomly deselect $N_{b}\left(r_{k}\right)-N_{b k}$ bonds from $\Theta_{i}$. These bonds will again be considered for subsequent $r_{k}$.

For an unpositioned bond $B$ in $\Theta_{k}$ with value $p_{b}$ of $P_{b}$ and length $l_{B}$, we calculate the value $p_{n}$ of $P_{n}$ based on the correlation between $P_{n}$ and $P_{b}$. From $O_{k}$, we select the subset of nodes $O_{k}^{\prime}$ with unused connections, i.e. for which the number of already connected bonds is less than its coordination number NC. Then, choose the node $\mathrm{N}_{1}$ in $O_{k}^{\prime}$ for which its $P_{n}$ value $p_{n 1}$ is closest to $p_{n}$. To choose a second node from $O_{k}^{\prime}$, an additional condition needs to be considered. Let $d_{1 j}$ be the Euclidian distance between the centres of $\mathrm{N}_{1}$ of radius $r_{n 1}$ and another node $\mathrm{N}_{j}$ of radius $r_{n j}$ in $O_{k}^{\prime}$. Then, determine the subset of candidate nodes $O_{k}^{\prime \prime}$ of $O_{k}^{\prime}$, for which $\left|d_{1 j}-r_{N_{1}}-r_{N_{j}}-l_{B}\right|<\omega d_{1 j}$, i.e. the distances to $\mathrm{N}_{1}$ is close to $l_{B}$. $\omega \varepsilon(0,1)$ is a predefined sufficiently small positive constant (we use $\omega=0.1$ ). If $O_{k}^{\prime \prime}$ is empty, select another $\mathrm{N}_{1}$ in $O_{k}^{\prime}$ and determine again the subset $O_{k}^{\prime \prime}$. Otherwise, select the second node $\mathrm{N}_{2}$ from $O_{k}^{\prime \prime}$ for which the $\mathrm{P}_{n}$ value $p_{n 2}$ satisfies $\left|p_{j}-p_{n}\right| \geq\left|p_{n 2}-p_{n}\right|$ for all other nodes $\mathrm{N}_{j}$ in $O_{k}^{\prime \prime}$. Finally, $\mathrm{N}_{1}$ and $\mathrm{N}_{2}$ are connected by the given bond $B$.

Similar to the above procedure, a boundary bond with length $l_{B}$ is positioned perpendicular to the inlet or outlet face and connected to a node $\mathrm{N}_{i}$ for which the distance $d_{B i}$ between its centre and the respective face is the closest to $l_{B}$.

\section{Validation of Stochastic Network Generation}

In this Section, we validate the statistical description and stochastic generation methods, based on two original networks extracted from the X-ray CT images of a Berea sandstone and a Castlegate sandstone sample. We use a state-of-the-art two-phase flow network model (Ryazanov et al. 2009), which is similar to the network model described by Valvatne and Blunt (2004), to calculate network permeabilities, capillary pressures and relative permeabilities. Since we do not compare the simulation results with data, but only compare different simulations using the same network model, we omit further description of the network model. First, we assess if the structural and flow properties of stochastically generated network models of the same size as the original $(\xi=1.0)$ are faithfully reproduced, and we show that ignoring certain correlations between the structural properties leads to incorrect flow predictions. Then, we investigate how the flow properties of generated networks of different sizes compare with those of the originally sized network. Finally, we consider the effect of the absolute size of the original network on the properties of the generated networks. In other words, we address the representativity of the original network.

\subsection{Rock Samples and Original Pore Networks}

Berea sandstone is relatively homogeneous, and is made up of well-sorted and well-rounded predominately quartz grains. Berea sandstone has been widely examined in the petroleum industry for many years in laboratory core flooding experiments. The Castlegate is a fine-tomedium-grained high-porosity sandstone made up of primarily quartz and rock fragments with small clay fractions. 
Table 3 The two original sandstone X-ray CT images with their characteristics (left), the extracted pore networks with some characteristics (including average node coordination number NC) and calculated permeabilities (middle) and extracted networks of the same size (right)

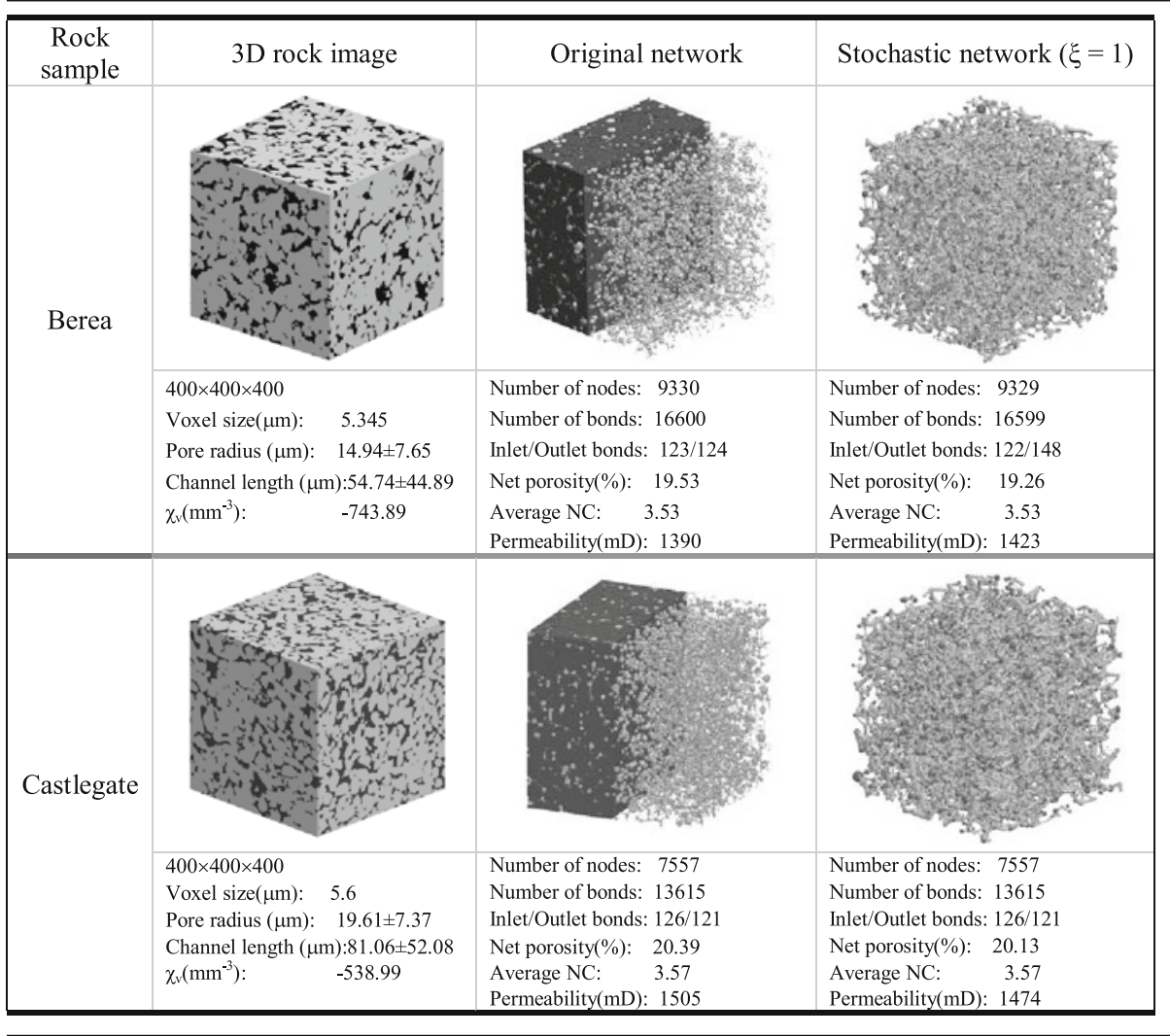

Table 3 shows the X-ray CT images of both sandstone samples and lists their major structural properties including porosities, pore radii, pore channel lengths and the specific Euler numbers $\left(\chi_{V}\right)$. Although the two samples have similar porosities, they differ significantly, as the Berea has smaller pore sizes and shorter pore channels, but it seems better connected than the Castlegate (lower $\chi_{V}$ ).

Furthermore, Table 3 shows the original networks extracted from the two images and also lists their major (average) properties. Contrary to the specific Euler numbers, the average coordination numbers of the two samples are very similar. Distributions of several properties (NR, NC, BS and BL) of both networks are presented in Figs. 6 and 7, as well as the respective connectivity functions. Observe from Table $1 \mathrm{~b}$ that the correlation structure for the Castlegate network is the same as for the Berea network, although the external correlation between $\mathrm{NR}$ and BR is now moderate. For the Berea network, the strong internal correlation between $\mathrm{NR}$ and NV and the strong external correlation between NR and BR, with their respective regression models are shown in Fig. $2 \mathrm{a}$ and $\mathrm{b}$. The conditional distribution between NV and NC was demonstrated in Fig. 4.

The drainage relative permeability and capillary pressure curves, assuming zero oil-water contact angles, for both networks are presented in Figs. 8 and 9. Note that the two networks 
(a)

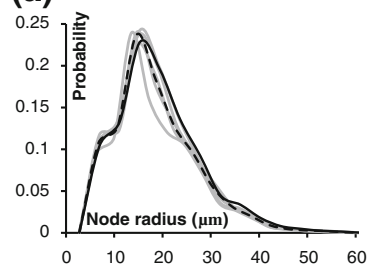

(d)

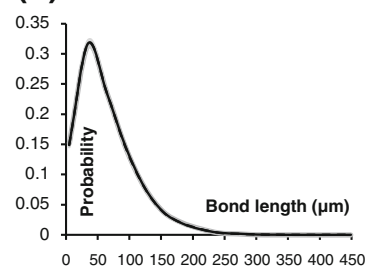

(b)

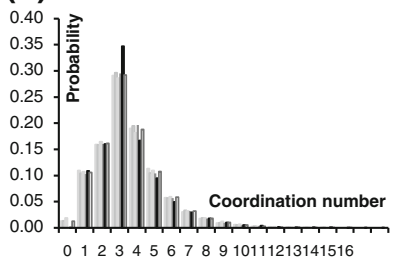

(e)

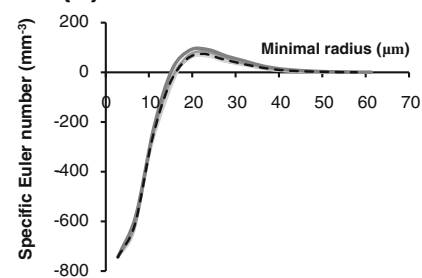

Fig. 6 Probability distributions of node radius (a), node coordination number (b), bond shape factor (c) and bond length (d), as well as the connectivity function (e) for the original (solid) and stochastic (dashed) Berea networks of the same size (i.e. $\xi=1$ ). Five stochastic realisations (grey curves) and their averages are shown

(a)

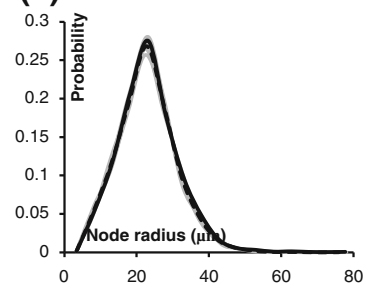

(d)

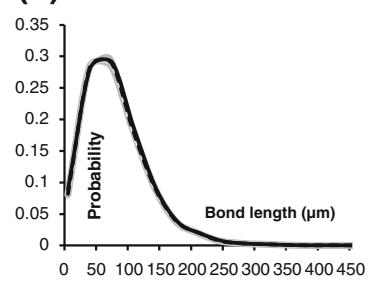

(b)

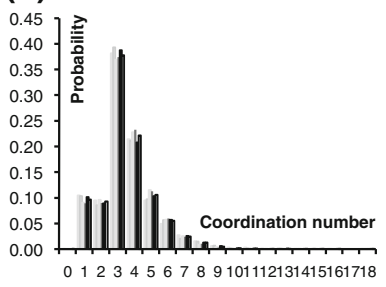

(e)

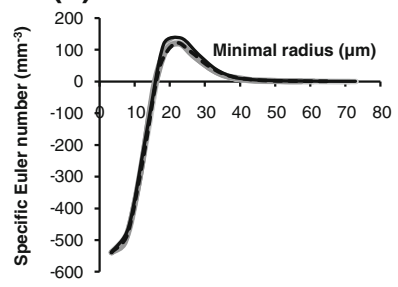

(c)

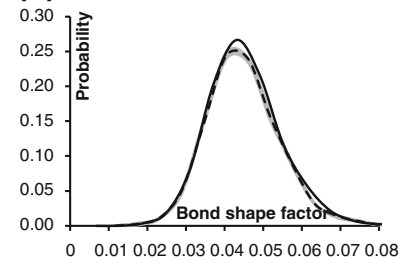


(a)

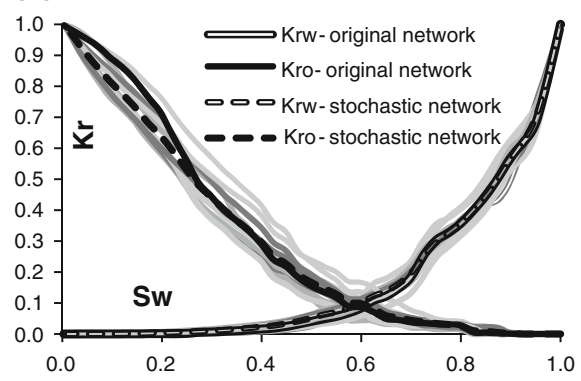

(b)

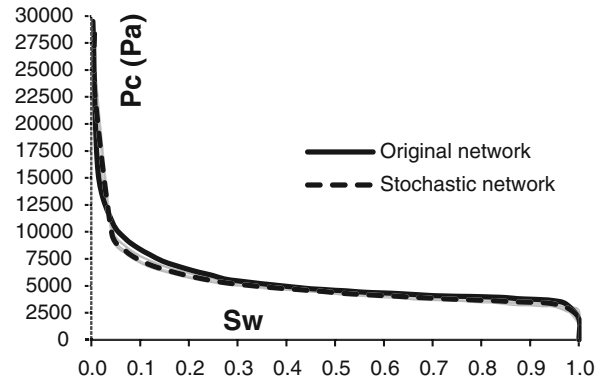

Fig. 8 Drainage relative permeability curves $\mathbf{a}$ and capillary pressure curves $\mathbf{b}$ of original Berea network (solid) and 10 stochastic networks (grey solid) with their average (dashed) for scaling factor $\xi=1$

(a)

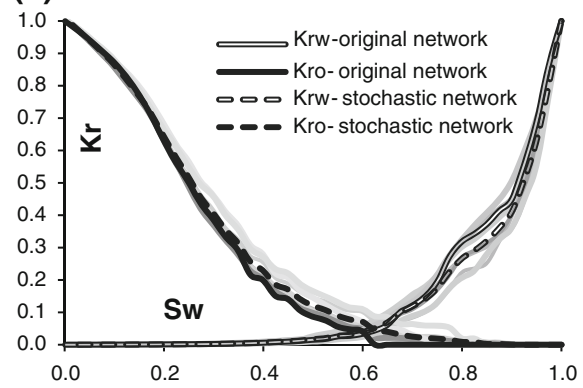

(b)

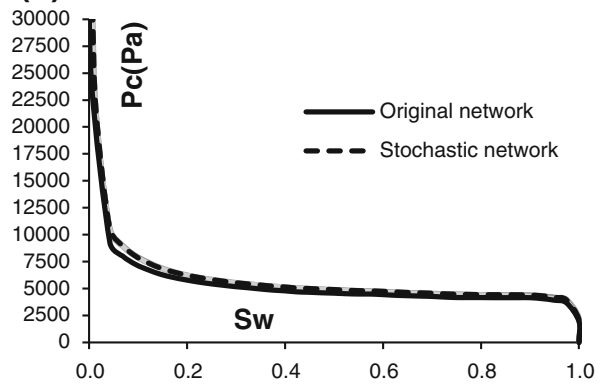

Fig. 9 Drainage relative permeability curves (a) and capillary pressure curves (b) of original Castlegate network (solid) and eight stochastic networks (grey solid) with their average (dashed) for scaling factor $\xi=1$

\subsection{Comparison of Original and Stochastic Networks}

We have generated stochastic networks of the same size $(\xi=1.0)$ from both the Berea and the Castlegate networks, and in the third column of Table 3, one realisation of each is shown. First, based on five realisations, we find that distributions of all geometrical and topological properties, of which a selection is shown in Fig. 6, show very good agreement with the distributions of the original network. Note, in particular, the close agreement of the NC (i.e. coordination number) distribution for the Berea network (Fig. 6b), which was generated from the NV distribution using a CPD (Fig. 4), which was in turn generated from the NR distribution using a regression equation (see Fig. 1). For the Berea network, we also demonstrate that the strong internal correlation between NR and NV, and the strong external correlation between NR and BR have been faithfully reproduced, as shown in Fig. 2a and b. These close agreements confirm that the statistics of the original network are representative; in other words, the sizes of the original networks were sufficient for stochastic generation. Moreover, the connectivity functions (Figs. 6e, 7e) have been accurately reproduced by the procedure described in Sect. 3.2, thus honouring the detailed topology of the network. The close agreement of the generated bond length distributions with the originals (Figs. 6d, 7d) demonstrates that the procedure for connecting nodes by bonds with lengths that closely match the distances between the nodes works satisfactorily. 


\section{(a)}

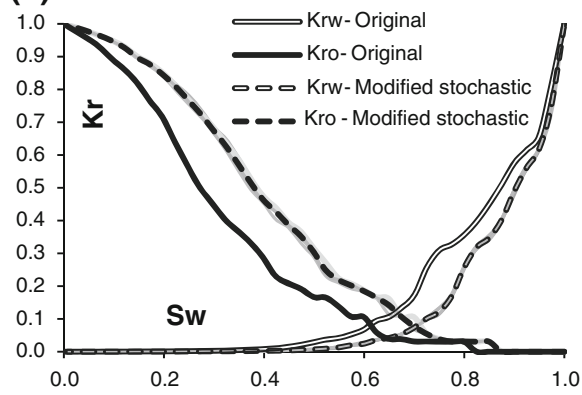

\section{(b)}

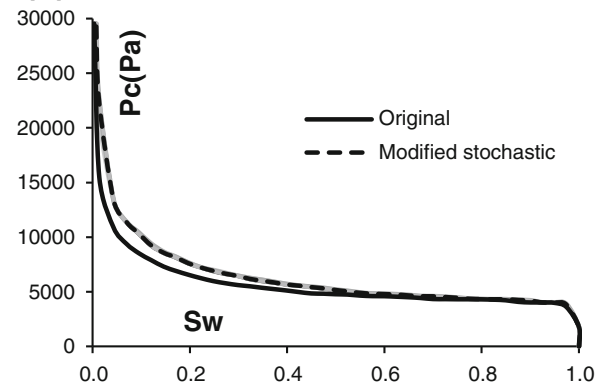

Fig. 10 Drainage relative permeability curves (a) and capillary pressure curves (b) of original Berea network (solid) and five modified stochastic networks (grey solid) with their averages (dashed), in which the internal correlation between NR and NV is ignored by randomly exchanging the volumes between pairs of nodes, while retaining all other properties.

Second, the absolute permeabilities of the original networks are well predicted by the stochastic networks (see Table 3). More importantly, in Figs. 7 and 8, we compare the relative permeability and capillary pressure curves for 10 stochastic realisations, with those for the original networks. We find that the averages of all curves compare very well with the originals, but the realisations for the relative permeability curves show some variability, in particular, the Kro curves. These matches are better than those for a Berea sandstone, obtained using a similar stochastic network generation technique, but without incorporation of the connectivity function (Idowu and Blunt 2008). The matches are also better than those for a soil sample, obtained using a generation technique based only on pore-size distribution and connectivity function on a regular lattice (Vogel and Roth 2001), although in this case, the comparison was made between stochastic networks and experiments on the underlying sample.

We have tested the sensitivity of the flow functions to some key correlations and to the connectivity function. In Fig. 10, we show the relative permeability and capillary pressure curves, for stochastic networks in which NV is uncorrelated and especially the strong correlation between NR and NV is ignored, but all other statistics are honoured. This was achieved by generating networks as before, while subsequently exchanging the volume values between pairs of nodes at random. Interestingly, the absolute permeability values are unaffected; however, Fig. 10 shows a systematic impact on the Pc and, in particular, the Kr curves. Similarly, if the external correlation between NR and BR is ignored (see Fig. 11), then by randomly exchanging pairs of bonds, the flow functions deviate systematically. Moreover, in this case also, the absolute permeabilities are different, i.e. on average, $1032 \mathrm{mD}$ as compared to 1423 $\mathrm{mD}$ for one of the unaltered generated networks. Finally, if we ignore the effect of the connectivity function by simply choosing only one interval of pore radii values (i.e. $m=2$ ) when incorporating the connectivity function (see Sect. 3.2), then the Pc and the Kro curves are strongly affected, but the Krw curve is only weakly affected, as shown in Fig. 12. Also the absolute permeability (averaged over five realisations) changed drastically to $798 \mathrm{mD}$.

Note that the large effect of ignoring the connectivity function, i.e. reduction of the absolute permeability, increase of Pc and decrease of Kro (see Fig. 12), points to a decrease in the effective pore radius (bond radius for drainage), i.e. the radius at which the porous medium percolates during drainage (e.g. O'Carroll and Sorbie 1993). This conjecture was confirmed by calculating the connectivity functions for the stochastic networks that do not honour the 
(a)

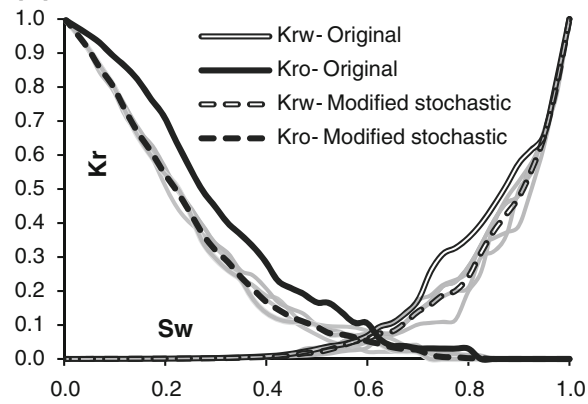

(b)

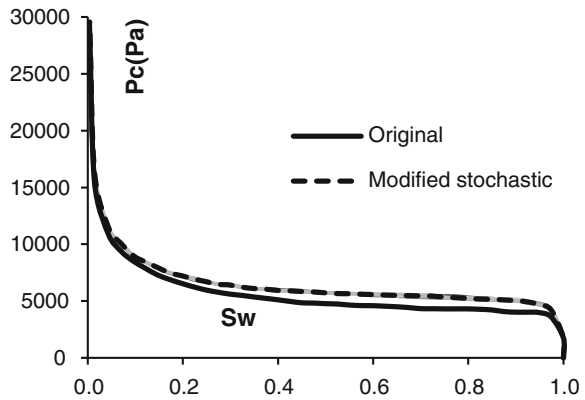

Fig. 11 Drainage relative permeability curves (a) and capillary pressure curves (b) of original Berea network (solid) and five modified stochastic networks (grey solid) with their averages (dashed), in which the external correlation between NR and BR is ignored by randomly exchanging pairs of bonds, while retaining all other properties.

(a)

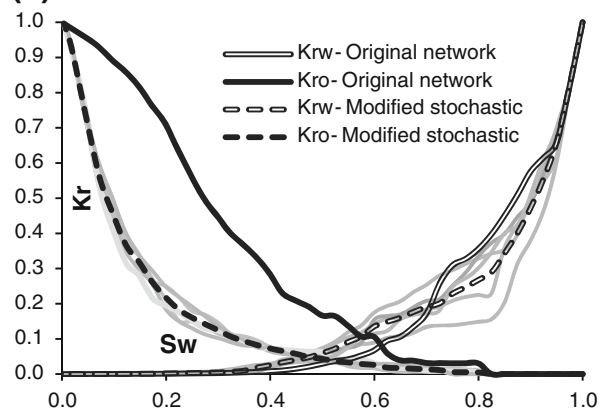

(c)

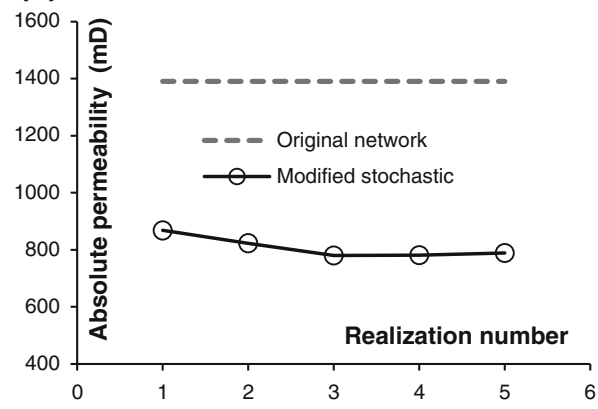

(b)

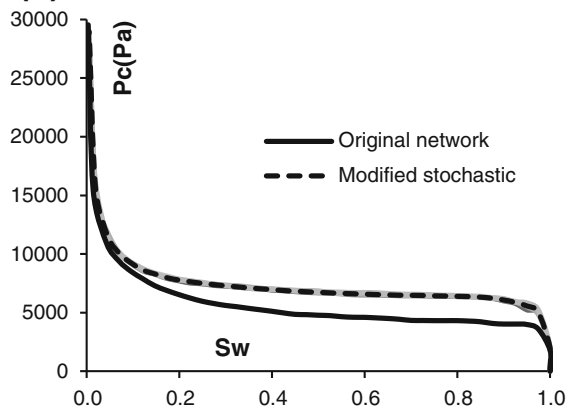

(d)

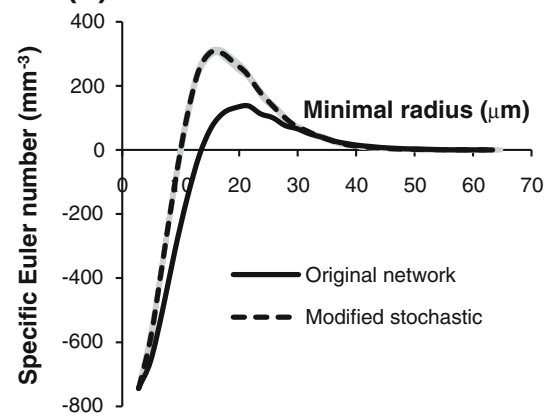

Fig. 12 Drainage relative permeability curves (a) and capillary pressure curves (b) of original Berea network (solid) and five modified stochastic networks (grey solid) with their averages (dashed), in which the connectivity function is not honoured, but only the coordination number distribution is matched. $\mathbf{c}$ shows the absolute permeability calculated from the original network and the five networks mentioned above. d shows the comparison between the resulting connectivity functions and the original connectivity function.

original connectivity function (see Fig. 12d). The zeros of these modified connectivity functions, which correspond closely to the percolation radii (Vogel and Roth 2001), were smaller than those in the networks with the proper connectivity functions. 


\section{(a)}

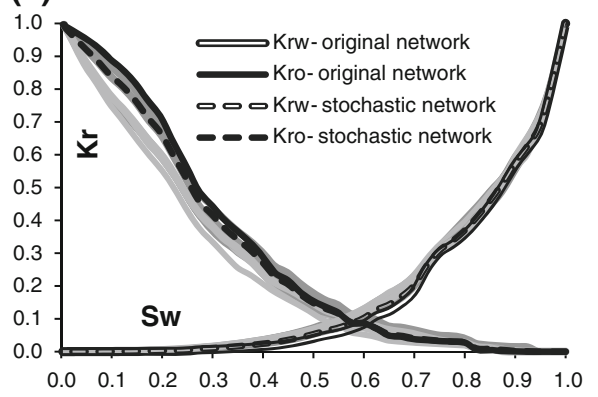

(b)

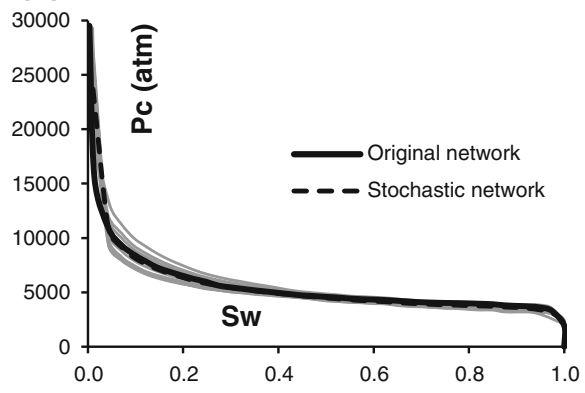

Fig. 13 Drainage relative permeability curves (a) and capillary pressure curves (b) of original Berea network (solid) and 10 stochastic networks (grey solid) with their average (dashed) for scaling factor $\xi=1.2$.

\subsection{Consistency Tests}

In the previous section, we have already demonstrated that for both the Berea and the Castlegate networks, our method succeeded in generating networks of the same size $(\xi=1.0)$ with structural and (multi-phase) flow properties that very well matched the properties of the originals. This indicates that the original networks are of representative volume, in the sense that the number of data points or, more specifically, the number of pore elements, is sufficient to capture the statistics of the network, for the reproduction of the flow properties. Moreover, our method has been successful in capturing the statistics of heterogeneities or spatial correlations at length-scales that are contained within the volumes of the original networks. The fact that, in particular, ignoring the external correlation between NR and BR or ignoring the connectivity function has led to significant deviations between generated and original flow properties (see Figs. 11, 12), indicate that these heterogeneities indeed exist.

To use our networks for the analysis of multi-scale networks or to validate our network generation method, we need to consider stochastic networks of arbitrary network size while all the structural features and physical properties of the corresponding original network are retained. For the two sandstone samples, a set of networks are generated of scaling factors ranging from $\xi=0.4$ to 2.0, three networks for each size, using the statistics of the two original networks shown in Table 3, which have scaling factor $\xi=1$.0. Table 4 shows that the smallest networks $(\xi=0.4)$ have only several hundred nodes and bonds, while the largest networks $(\xi=2.0)$ have around a hundred thousand nodes and bonds. The resulting average node coordination number (NC) and the predicted permeabilities are also presented in Table 4 . We find that for scaling factors $\xi$ significantly smaller than 1.0, the property values tend to deviate from the originals, as the number of pore element is no longer sufficient to realise the full statistics of the original networks. In addition, boundary effects will have increased for the permeability calculations. On the other hand, for scaling factors $\xi$ greater than 1.0 , the property values remain close to the originals as expected, since generated networks of $\xi=1.0$ already had representative volume. In addition, we present in Fig. 13, the multi-phase flow properties for a generated Berea pore network with scaling factor $\xi=1.2$. Average results are similar to those shown in Fig. 8 for $\xi=1.0$ as expected; however, the variability of the relative permeability realisations has decreased.

The above consistency should not only apply to our stochastic networks but also to the choice of the original rock image size. At a proper imaging resolution, larger rock samples are preferred to obtain sufficient information. However, for 3D image acquirement and image 
Table 4 Predicted permeabilities and some network characteristics for stochastic networks of increasing scaling factor $(\xi=0.4 \ldots 2.0)$ for the Berea and the Castlegate sandstone samples shown in Table 3

\begin{tabular}{lllllllllll}
\hline Rocks & Properties & \multirow{2}{*}{ Original network } & \multicolumn{8}{c}{ Network upscaling factor $\xi$} \\
\cline { 5 - 10 } & & & 0.4 & 0.6 & 0.8 & 1.0 & 1.2 & 1.4 & 2.0 \\
\hline \multirow{2}{*}{ Berea } & Volume $\left(\mathrm{mm}^{3}\right)$ & 9.773 & 0.625 & 2.111 & 5.003 & 9.773 & 16.887 & 26.817 & 78.183 \\
& Number of nodes & 9330 & 597 & 2015 & 4776 & 9329 & 16122 & 25601 & 74639 \\
& Number of bonds & 16600 & 1062 & 3585 & 8498 & 16599 & 28684 & 45549 & 132799 \\
& Average NC & 3.53 & 3.48 & 3.51 & 3.52 & 3.52 & 3.53 & 3.53 & 3.54 \\
& Perm (mD) & 1390 & 1704 & 1554 & 1518 & 1423 & 1491 & 1449 & 1409 \\
Castlegate & Volume $\left(\mathrm{mm}^{3}\right)$ & 11.239 & 0.729 & 2.427 & 5.754 & 11.239 & 19.421 & 30.841 & 89.915 \\
& Number of nodes & 7557 & 483 & 1632 & 3869 & 7557 & 13058 & 20736 & 60456 \\
& Number of bonds & 13615 & 870 & 2940 & 6970 & 13615 & 23526 & 37359 & 108920 \\
& Average NC & 3.57 & 3.52 & 3.54 & 3.56 & 3.57 & 3.57 & 3.58 & 3.58 \\
& Perm (mD) & 1505 & 1583 & 1591 & 1497 & 1474 & 1517 & 1477 & 1535 \\
\hline
\end{tabular}

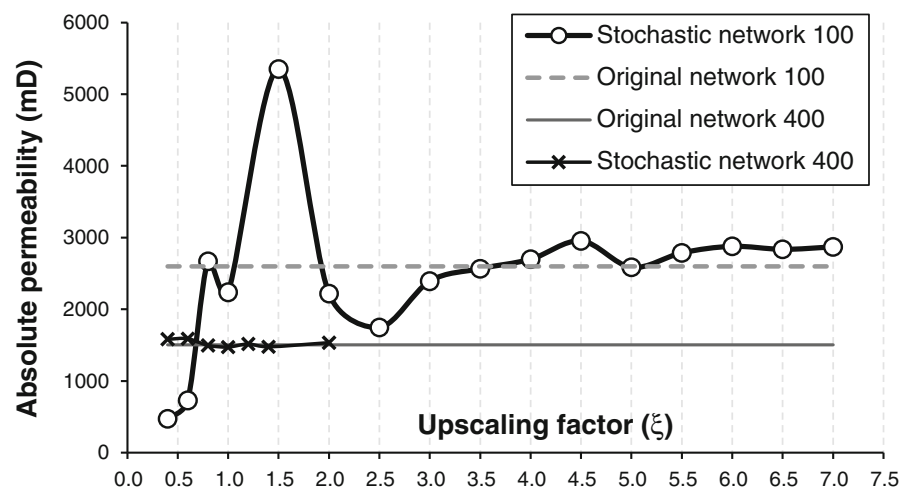

Fig. 14 Permeability predictions for networks of different scaling factors based on the network extracted from a small sub-image $\left(0.176 \mathrm{~mm}^{3}, 100^{3}\right.$ voxels, $\xi=1.0,2597 \mathrm{mD}$ (dotted grey)) cut out from the original image $\left(11.239 \mathrm{~mm}^{3}, 400^{3}\right.$ voxels, $1505 \mathrm{mD}$ (double-line) ) of the Castlegate sandstone sample shown in Table 3. The stochastic networks marked as 100 are based on the network extracted from the small sub-image, while those marked as 400 are based on the network extracted from the large image.

analysis, small rock samples are preferred. To test the minimum required sample size, we have cut out a sub-image of dimensions $100 \times 100 \times 100$ voxels from the Castlegate sandstone image shown in Table 3, i.e. 1/64 of the original volume from which we have extracted the pore network. Using this as the original network (with $\xi=1.0$ ), a series of stochastic networks were generated with network scaling factors $\xi$ ranging from 0.4 to 7.0 and corresponding volumes ranging from $0.011 \mathrm{~mm}^{3}$ to $60.236 \mathrm{~mm}^{3}$. The predicted permeability values for these stochastic networks are presented in Fig. 14. We find that the generated network of the same size as the subsample $(\xi=1.0)$ does not predict the permeability of its original $(2597 \mathrm{mD})$ and that only when the upscaling factor $\xi$ is sufficiently large, i.e. for $\xi>3.0$, the permeability value stabilizes. This demonstrates that the number of network elements of the subsample is too small. Furthermore, the converged permeability $(2776 \mathrm{mD})$ is very different from that for the original Castlegate sample $\left(11.23 \mathrm{~mm}^{3}\right)$ shown in Table 3 . This 
additionally indicates that the subsample does not capture the full statistics of the Castlegate sandstone. Therefore, an image of size greater than $100^{3}$ must be used to extract the original network on which the stochastic networks are to be generated. Based on Fig. 14 and Table 4, we suggest that the sample size of homogeneous sandstones, such as Berea and Castlegate, must be at least $2 \mathrm{~mm}^{3}$ to be representative $(\xi=0.6$ relative to the original sample size).

\section{Summary and Conclusions}

A new method has been developed to generate stochastic networks of arbitrary size representing the pore space in porous rocks. The method uses statistical information obtained from a pore network that is directly extracted from a 3D digital image of the pore space. The aim of this study is to create multi-scale networks by combining the statistics of networks at different length scales. First, the original network is characterised in terms of distributions of the geometrical pore properties (volume, radius, shape factor, etc.) and correlations between these properties for both network nodes and bonds, as well as the connectivity function describing the detailed network topology. Based on the correlation strengths, a structure of the dependencies between the various properties is identified. In general, strong correlations are described by regression equations, while weaker correlations are described by conditional probability distributions. Then, to create the stochastic network, the appropriate number of nodes and bonds are generated with the correlated properties of the original network. The nodes are randomly located in the given network domain and connected by bonds according to the strongest correlation between node and bond properties, while matching the bond lengths to the distances between nodes and honouring the connectivity function. The statistical description and stochastic network generation have been validated based on original networks extracted from the X-ray CT images of a Berea sandstone and a Castlegate sandstone sample, for which network permeabilities, as well as drainage capillary pressures and relative permeabilities, have been calculated.

For the given sizes of samples and corresponding networks, the structural property distributions, as well as the connectivity functions have been accurately reproduced in generated networks of the same size. In addition, excellent agreement has been obtained between the (average) calculated flow properties of the original and the generated networks, with some variability mainly around the relative permeabilities. The computation times for generating individual stochastic networks are very reasonable. For the two sandstone samples shown in Table 3 generating the stochastic networks with $\xi=1.0$ takes less than half the time of extracting the original networks from the rock images. Tests on generated networks in which one of the correlations have been ignored reveal the importance of each. Interestingly, ignoring the correlation between node radius and node volume, produced the correct absolute permeability but deviated multi-phase flow properties. Ignoring the correlation between node and bond radii and ignoring the connectivity function led to large deviations in all flow properties. This indicates that our method captures heterogeneities at length-scales contained within the network volumes.

Generating networks of different sizes, based on the original statistics, has revealed that, as expected, networks larger than the originals reproduced the flow properties of the original networks, contrary to significantly smaller networks, which had too few network elements to be statistically representative. Networks generated from the statistics of a smaller network extracted from a subsample of the original digital rock image were unable to reproduce the permeability of the subsample or the permeability calculated from the full sample. The latter indicated that the subsample was not representative. 
Open Access This article is distributed under the terms of the Creative Commons Attribution Noncommercial License which permits any noncommercial use, distribution, and reproduction in any medium, provided the original author(s) and source are credited.

\section{References}

Al-Kharusi, A.S., Blunt, M.J.: Network extraction from sandstone and carbonate pore space images. J. Pet. Sci. Eng. 56, 219-231 (2007)

Arns, J.-Y., Robins, V., Sheppard, A.P., Sok, R.M., Pinczewski, W.V., Knacksted, M.A.: Effect of network topology on relative permeability. Transp. Porous Med. 55, 21-46 (2004)

Bakke, S., Øren, P.E.: 3D pore-scale modeling of sandstone and flow simulations in pore networks. SPE J. 2, 136 (1997)

Bertrand, G.: Simple points, topological numbers and geodesic neighbourhoods in cubic grids. Pattern Recognit. Lett. 15, 1003-1011 (1994)

Biswal, B., Øren, P.-E., Held, R.J., Bakke, S., Hilfer, R.: Modeling of multiscale porous media. Image Anal. Stereol. 28, 23-34 (2009)

Blunt, M.J., Jackson, M.D., Piri, M., Valvatne, P.H.: Detailed physics, predictive capabilities and macroscopic consequences for pore-network models of multiphase flow. Adv. Water Res. 25, 1069-1089 (2002)

Coburn, T.C., Yarus, J.M., Chambers, R.L. (eds): Stochastic Modelling and Geostatistics: Principle, Methods, and Case Studies, vol. II. American Association of Petroleum Geologist (2007). ISBN: 0891817042

Delerue, J., Perrier, E.: DXSoil, a library for 3D image analysis in soil science. Comput. Geosci. 28, 10411050 (2002)

Idowu, N.A., Blunt M.J.: Pore-scale modeling of rate effects in Waterflooding. International Petroleum Technology Conference, IPTC/2008, 12292

Jensen, J.L., Lake, L.W., Corbett, P.W.M., Goggin, D.J.: Statistics for pertroleum Engineers and Geoscientists, 2nd edn. Elsevier (2000)

Jiang, Z.: Quantitative characterisation of the geometry and topology of the pore space in 3D rock images. $\mathrm{PhD}$ thesis, Institute of Petroleum Engineering, Heriot-Watt University, Edinburgh, UK (2008)

Jiang, Z., Wu, K., Couples, G.S, van Dijke, M.I.J., Sorbie, K.S., Ma, J.: Efficient extraction of networks from three-dimensional porous media. Water Resour. Res. 43, W12S03 (2007). doi:10.1029/2006WR005780

Jiang, Z., Wu, K., Couples, G.D., Ma, J.: The impact of pore size and pore connectivity on single-phase fluid flow in porous media. Adv. Eng. Mater. n/a. doi:10.1002/adem.201000255 (2010)

Lindquist, W.B., Lee, S.M., Coker, D.A., Jones, K.W., Spanne, P.: Medial axis analysis of void structure in three-dimensional tomographic images of porous media. J. Geophys. Res. 10, 8297-8310 (1996)

Lindquist, W.B., Venkatarangan, A., Dunsmuir, J., Wong, T.F.: Pore and throat size distributions measured from synchrotron X-ray topographic images of Fontainebleau sandstone. J. Geophys. Res. 24(2), 157177 (2000)

O'Carroll, C., Sorbie, K.S.: Generalization of the Poiseuille law for one- and two-phase flow in a random capillary network. Phys. Rev. E, 47, 3467-3476 (1993)

Pearson, K.: Notes on the history of correlation. Biometrika 13(1), 25-45 (1920)

Rodgers, J.L., Nicewander, W.A.: Thirteen ways to look at the correlation coefficient. Am. Stat. 42, 5966 (1988). doi: $10.2307 / 2685263$

Ryazanov, A.V., van Dijke, M.I.J., Sorbie, K.S.: Two-phase pore-network modelling: existence of oil layers during water invasion. Transp. Porous Med. 80, 79-99 (2009). doi:10.1007/s11242-009-9345-x

Sheppard, A.P., Sok, R.M., Averdunk, H.: Improved pore network extraction methods. In: Proceedings of the International Symposium of the Society of Core Analysts, Toronto, Canada (2005)

Silin, D.B., Jin, G., Patzek, T.W.: Robust determination of the pore space morphology in sedimentary rocks. J. Petrol. Technol. 56(5), 69-70 (2004)

Sok, R.M., Knackstedt, M.A., Sheppard, A.P., Pinczewski, W.V., Lindquist, W.B., Venkatarangan, A., Paterson, L.: Direct and stochastic generation of network models from tomographic images; effect of topology on residual staturations. Transp. Porous Med. 46, 345-372 (2002)

Strelen, J.C., Nassaj, F.: Analysis and generation of random vectors with copulas. In: Henderson, S.G., Biller, B., Hsieh, M.-H., Shortle, J., Tew, J.D., Barton, R.R. (eds.) Proceedings of the 2007 Winter Simulation Conference, pp. 488-496. Omnipress, Washington, DC (2007)

Valvatne, P.H., Blunt, M.J.: Predictive pore-scale modeling of two-phase flow in mixed wet media. Water Resour. Res. 40, W07406 (2004)

Vogel, H.J.: Morphological determination of pore connectivity as a function of pore size using serial sections. Eur. J. Soil. Sci. 48, 365-377 (1997) 
Vogel, H.J., Roth, K.: Quantitative morphology and network representation of soil pore structure. Adv. Water Res. 24, 233-242 (2001)

Vogel, H.J., Weller, U., Schluiter, S.: Quantification of soil structure based on Minkowski functions. Comput. Geosci. 36, 1236-1245 (2010) 